\title{
BMJ Open Impact of the COVID-19 pandemic on paediatric patients with cancer in low- income, middle-income and high-income countries: protocol for a multicentre, international, observational cohort study
}

To cite: Peter N,

Bandyopadhyay S, Lakhoo K, et al. Impact of the COVID-19 pandemic on paediatric patients with cancer in lowincome, middle-income and high-income countries: protocol for a multicentre, international, observational cohort study. BMJ Open 2021;11:e045679. doi:10.1136/ bmjopen-2020-045679

- Prepublication history and supplemental material for this paper is available online. To view these files, please visit the journal online (http://dx.doi. org/10.1136/bmjopen-2020045679).

NP and SB are joint first authors.

Received 08 0ctober 2020 Accepted 22 April 2021

D) Check for updates

(c) Author(s) (or their employer(s)) 2021. Re-use permitted under CC BY-NC. No commercial re-use. See rights and permissions. Published by BMJ.

Nuffield Department of Surgical Sciences, University of Oxford, Oxford, UK

Correspondence to

Dr Noel Peter;

noel.peter@ndorms.ox.ac.uk

\section{ABSTRACT}

Introduction Childhood cancers are a leading cause of non-communicable disease deaths for children around the world. The COVID-19 pandemic may have impacted on global children's cancer services, which can have consequences for childhood cancer outcomes. The Global Health Research Group on Children's Non-Communicable Diseases is currently undertaking the first international cohort study to determine the variation in paediatric cancer management during the COVID-19 pandemic, and the short-term to medium-term impacts on childhood cancer outcomes.

Methods and analysis This is a multicentre, international cohort study that will use routinely collected hospital data in a deidentified and anonymised form. Patients will be recruited consecutively into the study, with a 12-month follow-up period. Patients will be included if they are below the age of 18 years and undergoing anticancer treatment for the following cancers: acute lymphoblastic leukaemia, Burkitt lymphoma, Hodgkin lymphoma, Wilms tumour, sarcoma, retinoblastoma, gliomas, medulloblastomas and neuroblastomas. Patients must be newly presented or must be undergoing active anticancer treatment from 12 March 2020 to 12 December 2020. The primary objective of the study was to determine all-cause mortality rates of 30 days, 90 days and 12 months. This study will examine the factors that influenced these outcomes. $\chi^{2}$ analysis will be used to compare mortality between low-income and middle-income countries and high-income countries. Multilevel, multivariable logistic regression analysis will be undertaken to identify patient-level and hospitallevel factors affecting outcomes with adjustment for confounding factors.

Ethics and dissemination At the host centre, this study was deemed to be exempt from ethical committee approval due to the use of anonymised registry data. At other centres, participating collaborators have gained local approvals in accordance with their institutional ethical regulations. Collaborators will be encouraged to present the results locally, nationally and internationally. The results will be submitted for publication in a peer-reviewed journal.

\section{Strengths and limitations of this study}

- This is the first large-series, geographically comprehensive, multicentre, international cohort study to explore the management of childhood cancers in low-income, middle-income and high-income countries across the globe during the COVID-19 pandemic.

- The collaborative approach for this study allows a large series of high-quality data to be collected in a timely manner without overburdening high-volume, low-resource centres.

- A single study database will be used, which will allow for regular data analyses throughout the study in order to rapidly disseminate the emerging data.

- Medium-term impacts on childhood cancer outcomes will be assessed through a 12-month followup period.

- This study has limited its focus to nine of the the most common paediatric cancers globally as identified by the WHO and hence does not capture the effects of the pandemic on rarer cancers.

\section{INTRODUCTION}

The COVID-19 pandemic has taken a toll on our healthcare systems, economies and day-to-day lives. ${ }^{1}$ In order to combat the pandemic, multiple nations have restricted the services that their healthcare systems offer to their population. ${ }^{2-4}$ Lessons from the 2014-2016 Ebola epidemic suggest that these measures could lead to a rise in mortality and morbidity from other diseases. ${ }^{5}$ One particular group where the risk:benefit ratio of service disruption may have fallen on the wrong end of the spectrum is children with cancer, especially as children seem to be at lower risk of COVID-19 and its more severe manifestations. ${ }^{6}$

Childhood cancers have long been missing from the global child health agenda. 
Although children have curable cancers, they are often fatal without appropriate and timely diagnosis and treatment. In fact, childhood cancers are the second leading cause of non-communicable disease (NCD) deaths for paediatric patients around the world. ${ }^{7}$ Furthermore, there is a stark disparity of cancer survival rates between high-income countries (HICs) and low-income and middle-income countries (LMICs) with more than $90 \%$ of global childhood deaths from cancer occurring in LMICs. ${ }^{8}$ Current estimates suggest that children diagnosed with cancer in an HIC have a mean 5-year survival rate of $80 \%,{ }^{9-11}$ whereby children in LMICs have a mean 5 -year survival rate of $20 \% .{ }^{11}{ }^{12}$ Recent estimates put the incidence of childhood cancers in LMICs at almost 150 000 new cases per year; ${ }^{13}$ almost a half of all these cases go undiagnosed and untreated in Africa and Southeast Asia. ${ }^{14} 15$ The reason for this is multifactorial: delays in diagnosis, ${ }^{15} 16$ lack of access, ${ }^{13}$ poor investment into services $^{817}$ and inadequate support for workforce development. $^{8}$ The COVID-19 pandemic is likely to have placed an additional strain onto systems that have yet to be quantified and assessed. ${ }^{18}$

Hospital resource constraints, restructuring of services, and the lack of inpatient or community beds may have resulted in delays to treatment. Stay-at-home orders instituted by local governments may have affected the availability and affordability of transport, delaying access to treatment. In addition, a prevailing fear among parents regarding the infectious nature of the virus may have led some to avoid seeking treatment for their child. Attempts to mitigate the risk of infection may also have led to paediatric patients with cancer facing further delay in their treatment or a change to less effective therapeutic options. Furthermore, the socioeconomic impact of the pandemic may have resulted in a reduction of income, particularly among those who are informally employed in LMICs, which could have made treatment unaffordable. As such, there is a need to collect robust data about the changes that have occurred to paediatric cancer management, the factors that have driven this change, and the consequences for childhood cancer outcomes.

Studies have been launched that assess the effect of the COVID-19 pandemic on the outcomes of the adult oncological population ${ }^{19}$; however, there is a paucity of studies looking at the impact on the paediatric oncological population. ${ }^{20}$ There are little data available regarding the direct effects of COVID-19-as well as the measures imposed to minimise COVID-19 mortality and morbidity—on global children's cancer services, and hence the subsequent impact on outcomes for children with cancer.

We have designed and are running an international, multicentre study that will be performed prospectively and retrospectively to analyse and assess the impact of the COVID-19 pandemic on paediatric cancer services. The Global Health Research Group on Children's NonCommunicable Diseases (Global Children's NCDs) Collaborative consists of representations from the College of Surgeons of Southern, East and Central Africa, ${ }^{21}$ the
Global Children's Initiative for Surgery, ${ }^{22}$ the Pan-African Paediatric Surgical Association, ${ }^{23}$ the International Society of Paediatric Oncology ${ }^{24}$ and the International Society of Paediatric Surgical Oncology. ${ }^{25}$ These are all established global clinical and research groups that are renowned in their field with an extensive network of paediatric surgeons and oncologists globally. Furthermore, this study is also in collaboration with the Surgical Interventions Trial Unit (SITU) at the University of Oxford. SITU is one of seven Royal College of Surgeons of England specialist trial centres and consists of a multidisciplinary team with considerable methodological, study and trial expertise. Through this study, we aimed to investigate the changes that may have occurred to paediatric cancer management and the factors that have driven this change. Furthermore, we hope to examine the medium-term effect of the pandemic on childhood cancer outcomes globally. This pandemic has become the defining crisis of our generation, and its ramifications may stretch beyond the acute crisis and have far-reaching consequences for the future. Understanding its true impact, taking on key lessons and identifying vulnerabilities within health systems will help us develop solutions and prepare better for the future.

\section{Aims}

The primary aim was to undertake the first large-scale, geographically comprehensive, multicentre, international cohort study to determine the short-term and medium-term outcome of paediatric patients with cancer during the COVID-19 pandemic. The secondary aims were (1) to determine the variation in paediatric cancer management in LMICs and HICs across the globe during the COVID-19 pandemic and (2) to build global research capacity to empower individuals to conduct further research into children's NCDs globally.

\section{Objectives}

The primary objective of the study was to determine allcause mortality rates of 30 days, 90 days and 12 months in children with paediatric cancers during the COVID-19 pandemic across countries with low, middle and high Human Development Indices. This study will examine the factors that influenced these outcomes including tumourspecific data, patient-specific demographics and changes to health system frameworks. Secondary objectives of this study were to evaluate (1) the changes to paediatric cancer management during the COVID-19 pandemic; (2) the factors that influenced these changes from a health systems framework (eg, infrastructure, workforce, redeployment of staff, access to services, etc); (3) the infection rate of COVID-19 among paediatric patients' 30-day postcommencement anticancer treatment; and (4) the number of paediatric patients with cancer who were placed under palliative care or who sought abandonment of treatment ${ }^{26}$ during the pandemic. The fulfilment of these objectives will raise awareness and provide advocacy for paediatric patients with cancer within global health prioritisation, planning, policy and funding. 
Recruit collaborators

$\downarrow$

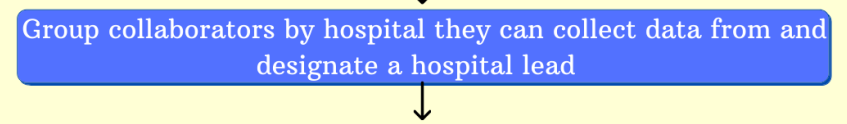

Obtain local study approval for each study site and

create a REDCAP account for the hospital lead

Hospital leads then share the REDCAP account with their respective teams

$\downarrow$

Identify eligible participants according to the eligibility criteria

$\downarrow$

Input data onto the REDCAP server

Figure 1 Step-by-step process for the formation of miniteams and the collection of local data. REDCap, Research Electronic Data Capture.

\section{METHODS AND ANALYSIS \\ Study design}

This is a multicentre, international, mixed (retrospective and prospective) cohort study that will use routinely collected hospital data in a deidentified and anonymised form. Patients will be recruited consecutively into the study, with a 12-month follow-up period. This will be followed by the data validation process. There will be no change in clinical care pathways. Participating hospitals will prospectively screen patients for eligibility to ensure that all patients fulfilling eligibility criteria are captured.

\section{Collaborators}

In accordance with National Research Collaborative authorship guidelines, ${ }^{27}$ all publication outputs will be listed under a unified corporate authorship: 'Global Children's NCDs Collaborative'. The generic collaborative method and the benefits that participants derive from it are based on previous well-documented evidence. ${ }^{28} 29$ Local collaborating investigators are required to establish mini-teams locally, gain study approval, use the protocol criteria to appropriately identify patients for study inclusion, and collect data and upload it to the Research Electronic Data Capture (REDCap) web application (figure 1). There are no limits to the size of the team at each site, as it should be based around local capacity and service demands. Each mini-team will have a local study lead who will hold overall responsibility for ensuring the data for their hospital are accurate, complete and without duplications. Each mini-team must contain at least one senior clinician to oversee the data collection process. All collaborators from sites who contribute at least one patient will be recognised on any resulting publications as PubMed-citable collaborative authors in accordance with the roles defined as follows:

Writing group: responsible for the overall scientific content, data analysis and preparation of research manuscripts

Steering committee: responsible for the protocol design, project coordination and data handling.
Operational committee: responsible for the day-to-day operational management to ensure the study remains on track in delivering its objectives.

Research capacity building committee: responsible for ensuring the fulfilment of the research capacity building side of the study.

Country leads: responsible for recruiting other collaborators from within their country and for providing advice and support regarding gaining local study approval and data collection.

Hospital leads/data collectors: responsible for the running of the study locally.

\section{Hospital inclusion criteria}

Any hospital or cancer centre providing anticancer treatment to paediatric patients will be eligible to partake in this study. Anticancer treatment is defined as any intervention to treat, cure, debulk and provide symptomatic relief for the patient's cancers through the modalities of surgery, radiotherapy, chemotherapy or immunological therapy.

\section{Patient inclusion criteria}

Patients will be included if they are below the age of 18 years and undergoing anticancer treatment for the following clinically diagnosed cancers: acute lymphoblastic leukaemia, nonHodgkin's lymphoma, Hodgkin lymphoma, Wilms tumour, rhabdomyosarcoma, osteosarcoma, Ewing's sarcoma, retinoblastoma, gliomas, medulloblastomas and neuroblastomas. ${ }^{30}$ These represent a selection of the the most common paediatric cancers globally as identified by the WHO. ${ }^{31}$ Patients must be newly presented or be undergoing active anticancer treatment from the start of the COVID-19 pandemic (as declared by the WHO on 12 March 2020) to 12 December 2020 (figure 2). Given that the COVID-19 pandemic may have already peaked in some regions, retrospective data collection will also be permitted if collaborators are able to identify eligible patients that were either newly presented or undergoing active treatment from the start of the pandemic.

\section{Patient exclusion criteria}

All patients who are 18 years and above will be excluded from the study. Patients will be excluded if they do not have paediatric cancer diagnosis set out within the inclusion criteria. Patients will be excluded if they newly present after 12 December 2020.

\section{Data collection}

Data collected will include baseline demographic data, tumour-specific data, timelines for dates of diagnosis, factors behind any change in management during the pandemic, operative and non-operative cancer-related treatments, treatment related to COVID-19 (where applicable), pathology and clinical outcome data (online supplemental appendix S1 and table 1).

Data from the study will be collected and stored online through a secure server running the REDCap web application. REDCap allows collaborators to enter and store data in a secure, encrypted system. The REDCap database used for this study will be within the University of Oxford 


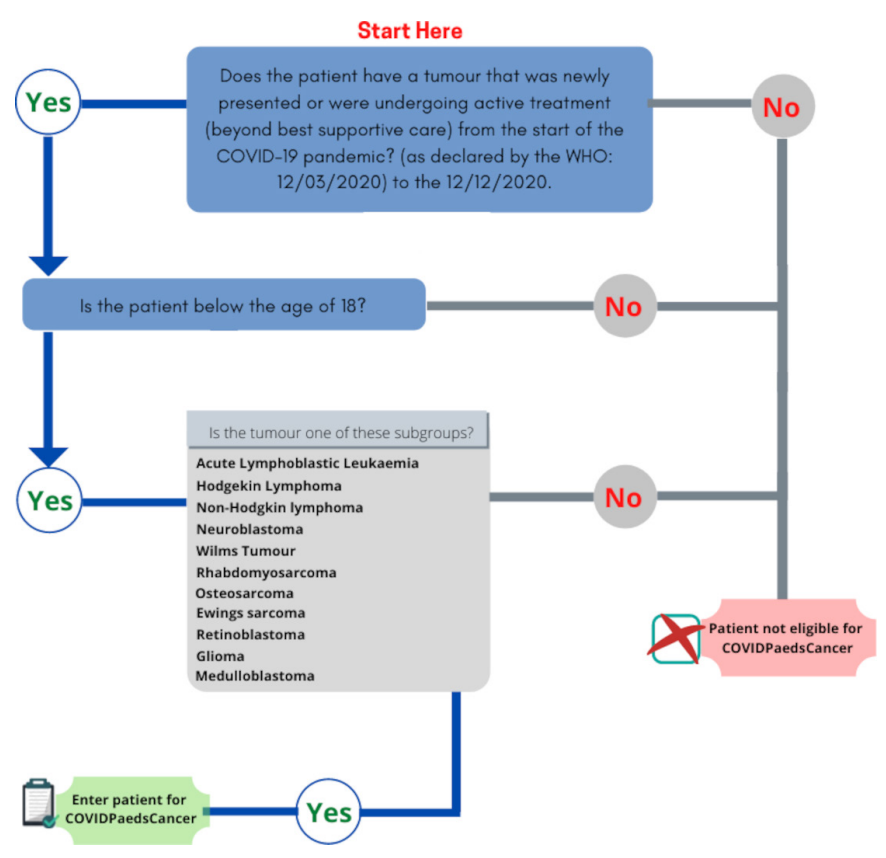

Figure 2 Patient inclusion and exclusion criteria flow diagram.

Virtual Machine architecture, which is physically secured. The security of the study REDCap database system is governed by the policies of the University of Oxford, UK, in accordance with the requirements of the General Data Protection Regulations. Only authorised members of the local data collection team and steering committee will have access to the research data.

A single study database will be used for all hospitals participating in this study. This will allow us to perform regular data analyses throughout the study in order to rapidly disseminate the emerging data. No sensitive or identifiable data will be collected on the database; each hospital team will only upload anonymised data. The central research team will not have any access to patient identifiable data. All data will be analysed and reported in summary format. No individual will be identifiable. The anonymised data generated by the study will be held centrally at the University of Oxford, UK.

\section{Data quality}

To ensure high quality of data, a detailed protocol was sent to all collaborators. The protocol was translated to a local language when needed to improve clarity. This was done with the assistance of a native speaker to assist with accuracy. Clear and concise definitions were provided on the data collection forms and within REDCap when entering the data. Virtual monthly meetings have been ongoing between the steering committee and all local study leads to address any challenges and issues that may have arisen.

In addition, the steering committee meets twice a week on a virtual platform to discuss and resolve issues arising from the study. Following these discussions, a frequently asked questions document is circulated via email biweekly. There exists an operational team with members across nine countries, who manage the day-to-day running of the study. This is further supported with our collaboration with SITU, which have a vast multidisciplinary experience and expertise in delivery of large multicentre studies.

\section{Data validation}

Data validation will be in two parts. First, hospitals will selfreport the key processes used to identify and follow-up patients. Second, independent validators will quantitatively report case ascertainment and sampled data accuracy. This process will involve $10 \%$ of collaborating centres being selected randomly for data validation. An additional independent research collaborator at each validation centre will determine the number of patients eligible for study inclusion within the data collection period, to check if any were missed, and to collect a selection of the data again to be checked for accuracy. The independent collaborator will be identified and invited to participate in the study lead at the allocated centre. They must be a healthcare professional within the team who cares for children with the study conditions, but who was not involved in the initial data collection. The validation data will be collected on a separate REDCap validation database and the inputted data will be cross-checked with that entered into the main database to assess for accuracy. A weighted kappa statistic will be used to determine the level of agreement between the study patient data and the validation patient data. This will be presented as a proportion of agreement for each variable being validated.

\section{Sample size calculation}

Children diagnosed with cancer in an HIC have been reported to have a 12-month mortality of $10 \%$ prior to the COVID-19 pandemic. A child diagnosed with cancer in a LMIC was known prior to the COVID-19 pandemic to have four times the relative risk of death compared with a child in a HIC. ${ }^{11}$ Studies on the impact of COVID-19 on cancer in adults have found that individuals in HICs with certain types of cancers have had up to a $10 \%$ increase in their absolute risk of mortality. ${ }^{32} 33$ This finding has been extrapolated to the paediatric population for sample size calculation purposes. This finding has yet to be replicated in the LMIC setting. Assuming the COVID-19 pandemic has not increased the risk of death in children in LMICs, children in HICs and LMICs are anticipated to have a 12-month mortality of $20 \%$ and $40 \%$, respectively (these assumptions maximise the sample size needed, reducing the risk of the study being underpowered). ${ }^{34}$ Less than $20 \%$ of all paediatric cancers are found in HICs. Therefore, an enrolment ratio of 4:1 between LMICs and HICs is expected. Using a two-sided test at the $1 \%$ significance level, a sample size of 490 patients would enable us to detect a $20 \%$ absolute difference in 12-month mortality between LMICs and HICs with a power of at least $90 \%$. Of these patients, 392 would be from LMICs. Allowing for approximately $20 \%$ loss to follow-up, the sample size needed is 650 patients with 500 patients from LMICs. 
Table 1 Key questions present in data collection form

\begin{tabular}{ll}
\hline REDCap sheet & Key questions \\
\hline Baseline information & Age of patient (in years) \\
& Does the patient have a tumour? \\
& Sex \\
& Weight $(\mathrm{kg})$ \\
& ASA
\end{tabular}

Diagnostic group/subgroup of tumour

REDCap field and notes

Patient's age in years

Select yes if the patient has a tumour, no otherwise

Patient sex

The first weight of the patient taken during admission

ASA at time of surgery

Full definitions are available online (https://www.asahq.org/standards-and-guidelines/asaphysical-status-classification-system)

Select the type of tumour present in the patient.

Acute lymphoblastic leukaemia-cancer of white blood cells

Hodgkin lymphoma; full definitions are available online (https://www.mayoclinic.org/ diseases-conditions/hodgkins-lymphoma/symptoms-causes/syc-20352646)

Non-Hodgkin lymphoma; full definitions are available online (https://www.nhs.uk/conditions/ non-hodgkin-lymphoma/)

Neuroblastoma-cancer of immature nerve cells

Wilms tumour; full definitions are available online (https://www.mayoclinic.org/diseasesconditions/wilms-tumor/symptoms-causes/syc-20352655\#: :text=Wilms' tumour is a rare,less common after age 5)

Rhabdomyosarcoma; full definitions are available online (https://www.mayoclinic.org/ diseases-conditions/rhabdomyosarcoma/symptoms-causes/syc-20390962) Osteosarcoma-a type of bone cancer

Ewings sarcoma; full definitions are available online (https://www.mayoclinic.org/diseasesconditions/ewing-sarcoma/symptoms-causes/syc-20351071)

Retinoblastoma-a type of cancer that develops in the retina of the eye

Glioma-a tumour that develops in the central nervous system

Medulloblastoma-a malignant tumour that starts in the cerebellum

Grade of glioma

Staging

Date of diagnosis

What was the initial multi-disciplinary team (MDT)/tumour board decision for managing this tumour?

Date of treatment decision by the tumour board

Would this decision have been different prio to the COVID-19 pandemic?

What would the pre-COVID-19 decision for managing this tumour be?

Did the patient have chemotherapy during
the study window?
Did the patient have chemotherapy during
the 90 -day follow-up period?
Is there still a plan for chemotherapy
treatment?
Were there any changes to the
chemotherapy treatment due to the
COVID-19 pandemic?
What were the reasons for the change(s) to
the treatment?

If glioma is chosen, you will be asked to select one of the following: Low grade (WHO grade I/II) (from astrocytes and oligodendrocytes) High grade (WHO grade III/IV) (malignant tumours that develop from astrocytes) Unknown

Full definitions are available online (https://cancerqld.blob.core.windows.net/content/docs/ childhood-cancer-staging-for-population-registries.pdf) ${ }^{37}$

Depending on the type of tumour selected, a staging system will appear.

Full definitions of each stage are available online (https://cancerqld.blob.core.windows.net/ content/docs/childhood-cancer-staging-for-population-registries.pdf) ${ }^{37}$

The date at which the patient was clinically diagnosed to have the tumour type selected earlier The date of diagnosis can precede the start date for this study.

The initial treatment plan for the patient; it can involve a combination of chemotherapy, radiotherapy, immunological therapy and surgery. Please select no anticancer therapy only if the other four options are not selected.

Date at which the initial treatment plan for the patient was made

If the date at which the initial treatment plan for the patient was made prior to the pandemic, please select no. Otherwise, please review hospital protocol and contact senior clinicians to assess if the treatment plan would have been different for the patient if the initial treatment plan were made prior to start of the pandemic.

Please select the treatment plan for this patient that would have been opted for prior to COVID-19. It can involve a combination of chemotherapy, radiotherapy, immunological therapy, and surgery. Please select no anticancer therapy only if the other four options are not selected.

Please select yes if the patient had chemotherapy between 12 March 2020 and 12 December 2020 inclusive. This is regardless of if there was an initial plan to treat with chemotherapy.

Please select yes if the patient had chemotherapy within 90 days of their first presentation to the hospital within the study period (between 12 March 2020 and 12 December 2020). This is regardless of if there was an initial plan to treat with chemotherapy.

Please select yes if chemotherapy is planned for this patient. This is regardless of if there was an initial plan to treat with chemotherapy.

Please select 'No change to chemotherapy care because of COVID-19' if there were no changes made to chemotherapy plans due to COVID-19 (including if there were changes made due to other non-COVID-19-related issues). Otherwise, select the correct options from the choice provided.

Please select as many reasons as appropriate that explain the change(s) to chemotherapy treatment. 
Table 1 Continued

\begin{tabular}{lll}
\hline REDCap sheet Key questions $\quad$ REDCap field and notes
\end{tabular}

Radiotherapy Did the patient have radiotherapy during the study window?

Please select yes if the patient had radiotherapy between 12 March 2020 and 12 December 2020. This is regardless of if there was an initial plan to treat with radiotherapy.

Did the patient have radiotherapy during the Please select yes if the patient had radiotherapy within 90 days of their first presentation to 90-day follow-up period? the hospital within the study period (between 12 March 2020 and 12 December 2020). This is regardless of if there was an initial plan to treat with radiotherapy.

Is there still a plan for radiotherapy treatment?

Please select yes if radiotherapy is planned for this patient. This is regardless of if there was an initial plan to treat with radiotherapy.

Were there any changes to the radiotherapy treatment due to the COVID-19 pandemic?

Please select 'No change to radiotherapy care because of COVID-19' if there were no changes made to radiotherapy plans due to COVID-19 (including if there were changes made due to other non-COVID-19-related issues). Otherwise, select the correct options from the choices provided.

What were the reasons for the change(s) to the treatment?

What was the radiation field? treatment.

Whole brain and spinal cord radiation therapy (craniospinal radiation): radiation given to the whole brain and spinal cord.

Focal (brain): radiation not given to the whole brain and spine cord

Local: radiation directed towards a specific site

Wide-field: radiation that is not directed towards a specific single site

Radiotherapy approach

Please select either photon or proton beam therapy. You can find more information about either therapy online (https://breast360.org/topic/2015/01/01/radiation-therapy-photons-vsprotons/).

Immunological therapy Did the patient have immunotherapy during
the study window?

Please select yes if the patient had immunological therapy between 12 March 2020 and 12 December 2020. This is regardless of if there was an initial plan to treat with immunological therapy.

Did the patient have immunotherapy during Please select yes if the patient had immunological therapy within 90 days of their first the 90 -day follow-up period?

Is there still a plan for immunotherapy treatment? presentation to the hospital within the study period (between 12 March 2020 and 12 December 2020). This is regardless of if there was an initial plan to treat with immunologica therapy

Please select yes if immunological therapy is planned for this patient. This is regardless of if there was an initial plan to treat with immunological therapy.

Were there any changes to the immunotherapy treatment due to the COVID-19 pandemic?

Please select 'No change to immunotherapy care because of COVID-19' if there were no changes made to immunological therapy plans due to COVID-19 (including if there were changes made due to other non-COVID-19-related issues). Otherwise, select the correct options from the choices provided.

What were the reasons for the change(s) to the treatment?

Please select as many reasons as appropriate that explain the change(s) to immunological therapy treatment.

Surgery Did the patient have surgery during the study window?

Did the patient have surgery during the 90day follow-up period?

Date of surgery

Is there still a plan for surgical treatment?

Were there any changes to the surgical treatment due to the COVID-19 pandemic?

What were the reasons for the change(s) to the treatment?

What type of hospital was the operation performed in?

Time from admission to operation (preoperative delay)

Urgency of surgery 


\begin{tabular}{|c|c|c|}
\hline REDCap sheet & Key questions & REDCap field and notes \\
\hline \multirow[t]{4}{*}{$\begin{array}{l}\text { No anticancer } \\
\text { treatment }\end{array}$} & $\begin{array}{l}\text { Did the patient or their family choose to } \\
\text { avoid treatment during the pandemic before } \\
\text { the initial MDT (tumour board) meeting? }\end{array}$ & $\begin{array}{l}\text { Please select yes if the patient or their family choose to not take anti-cancer therapy before } \\
\text { a decision of whether to treat with anti-cancer therapy had been made. }\end{array}$ \\
\hline & $\begin{array}{l}\text { Did the patient have palliative treatment } \\
\text { during the study window? }\end{array}$ & $\begin{array}{l}\text { Please select yes if the patient had palliative treatment between the } 12^{\text {th }} \text { of March } 2020 \text { and } \\
\text { the } 12^{\text {th }} \text { of December } 2020 \text {. This is regardless of if there was an initial plan to treat with anti- } \\
\text { cancer therapy }\end{array}$ \\
\hline & $\begin{array}{l}\text { Were there any changes to the } \\
\text { immunotherapy treatment due to the } \\
\text { COVID-19 pandemic? }\end{array}$ & $\begin{array}{l}\text { Please select 'No change to palliative care because of COVID-19' if there were no changes } \\
\text { made to palliative treatment plans due to COVID-19 (including if there were changes made } \\
\text { due to other non-COVID-19-related issues). Otherwise select the correct options from the } \\
\text { choices provided. }\end{array}$ \\
\hline & $\begin{array}{l}\text { What were the reasons for the change(s) to } \\
\text { the treatment? }\end{array}$ & $\begin{array}{l}\text { Please select as many reasons as appropriate that explain the change(s) to palliative } \\
\text { treatment. }\end{array}$ \\
\hline \multirow[t]{4}{*}{ Outcomes } & $\begin{array}{l}\text { Complications within } 30 \text { days from their first } \\
\text { anticancer treatment post-11 March } 2020 ?\end{array}$ & $\begin{array}{l}\text { Please select 'No complication' if there were no complications due to the anti-cancer } \\
\text { treatment. Otherwise select the correct options from the choices provided. }\end{array}$ \\
\hline & Outcomes at 30-day follow-up? & $\begin{array}{l}\text { Please select whether the patient is dead or alive. Please use the descriptors in the answers } \\
\text { below to guide you in selecting the answer: } \\
\text { Died-did not receive anticancer treatment } \\
\text { Died-during anticancer treatment } \\
\text { Died-on days } 0-7 \text { after anticancer treatment } \\
\text { Died-on days } 8-30 \text { after anticancer treatment } \\
\text { Alive-remains admitted in hospital } \\
\text { Alive-transferred to another hospital } \\
\text { Alive-discharged to a rehabilitation centre } \\
\text { Alive-discharged home }\end{array}$ \\
\hline & Mortality at 90-day follow-up? & Please select whether the patient is dead or alive. \\
\hline & Mortality at 12-month follow-up? & Please select whether the patient is dead or alive. \\
\hline
\end{tabular}

ASA, American Society of Anesthesiologists; REDCap, Research Electronic Data Capture.

\section{Data analysis}

Analysis will be performed according to the Strengthening the Reporting of Observational Studies in Epidemiology guidelines for observational studies. ${ }^{35}$ Data will be analysed using STATA and SAS V.9.4. Duplicates will be removed if present. Missing data for the covariates will be analysed to determine whether it is related to the outcome and either complete-case analyses or multiple imputation techniques will be used for analyses accordingly. A complete-case analysis technique will be used to handle missing outcome data. Significant differences in 30-day, 90-day and 12-month mortality between LMICs and HICs will be determined for each of the study conditions using $\chi^{2}$ analysis, or Fisher's exact test if either group contains less than 10 patients. World Bank classification of LMICs and HICs during the fiscal year 2021 will be used. ${ }^{36}$ Multivariate logistic regression analyses will be conducted between covariates and the primary outcome of mortality. The least absolute shrinkage and selection operator (LASSO) method will be used for variable selection, and to determine the final multilevel multivariable logistic model of covariates affecting outcomes. Results will be presented as ORs with corresponding 95\% CIs. The intent of this model is to simply explain relationships between various factors and mortality. A multilevel, discrete time model will also be undertaken to identify patient-specific demographic data, cancer-specific data, pandemic-specific data (eg, COVID-19 infection), and institutional and health system factors affecting timing of death with adjustment for confounding factors. Analysis will be stratified according to whether COVID-19 was confirmed, and results will be presented as ORs with $95 \%$ CIs. Sensitivity analyses will be carried out, including only patients with laboratory-confirmed COVID-19 infection.

\section{Patient and public involvement}

Patients and members of the public actively participated in the preparation and formulation of this proposal. A preliminary meeting between the steering committee and parents of children with cancer was organised. Parents of 11 children from various continents (North America, Europe, Asia and Africa) took part in this meeting. Their children had a range of neoplasms that included leukaemia, rhabdomyosarcoma, osteosarcoma and Wilms tumour. The treatments received comprised chemotherapy with or without surgery, and one case also required blood transfusions. It was found that $40 \%$ of the children represented in the group had been impacted by COVID-19 in one of three key ways. First, follow-up clinics had become virtual. Second, there were delays in surgery. Last, the additional rules during the pandemic meant that parents had to receive news from doctors on their own, without their partners. All parents agreed on the value and benefit of the study. Two parents (one from the UK and one from Nigeria) agreed to be contacted for their input on the findings and dissemination of the results.

\section{Research capacity building}

Participation in the study will provide collaborators with experience of undertaking research including gaining local study approval, using a protocol to identify patients and collect data, use of the REDCap data collection tool, the process of data validation and an example of data analysis, 
interpretation and write-up. An online training session on how to set up a project using REDCap will be offered to all collaborators who are interested in undertaking their own study using this software. Through this process, we hope to support the enhancement of research capacity among the collaborating team, which in turn aims to encourage further research into childhood NCDs globally.

In addition to the aforementioned data, collaborators will have the opportunity to undertake an optional research training fellowship that is running alongside the main study. During this fellowship, the aim will be for collaborators to develop and undertake their own local research project. Monthly research webinars will be provided covering the following topics:

1. Generation of a research question and hypothesis.

2. Types of study design.

3. Protocol writing.

4. Ethical considerations and study approval.

5. Data collection.

6. Data cleaning and analysis.

7. Data interpretation.

8. Preparing an abstract for submission to a conference for presentation.

9. Writing a manuscript.

10. Choosing a journal and submitting for publication.

In total, there will be 10 online sessions over 12 months starting in October 2020. Each session will last between 1 and 2 hours. Each stage of the development and undertaking of the main study will be used as a working example during the sessions. Webinars will be undertaken in English, but a summary of each session will be provided in multiple languages as required. In conjunction with the webinars, a mentoring scheme will be established where collaborators are partnered with an academic to provide one-on-one advice and support throughout the development, undertaking and write-up of their study. The aim will be for each participant to produce their own abstract for submission to a conference for publication. Mentors will also support participants to write up their results for publication. All participants will receive a certificate to confirm completion of the research training fellowship.

\section{ETHICS AND DISSEMINATION}

\section{Research ethics approval}

The conduct of the study will conform to the principles of the Declaration of Helsinki (last revised 2013). Furthermore, the study will be conducted in accordance with International Conference on Harmonisation Guideline for Good Clinical Practice.

Despite having a research objective, the study was exempt from ethical committee approval at the host institution due to the study being based on anonymised medical record data (online supplemental appendices S2 and S3). The local study lead at each participating site is responsible for gaining approval to participate in the study at their institution according to their local ethical regulations. Local approvals should cover inclusion of all cancer types within this study. Collaborators are required to confirm that relevant local approval is in place at the time of uploading each patient record to the study database. Data transfer agreements were legally signed between institutions where required. Study documents were translated into the local language where required.

We have a team of 10 individuals within the operational committee who offer weekly support services for institutional review board ethics applications across all hospital sites. During these virtual sessions, collaborators and hospital site leaders meet to discuss challenges they may have faced with any element of the study, including gaining local approvals.

\section{Dissemination}

The results generated from this study will be fed back to the local workforce in each country, as it has direct implications for clinical practice and health system organisation around the world. Health system planners and local clinicians should be able to use our data and the analysis performed on it to make the necessary adjustments needed to balance the risks associated with COVID-19 infection against the risk of altering paediatric management at a population level and an individual level. Data will be fed back in real time to optimise the care of paediatric patients with cancer globally throughout the course of the study. In addition, local healthcare workers internationally will be collecting the data and communicating regularly with one another. This will allow for the sharing of ideas and best practices that could be modified and implemented in local settings.

Intermittently, the results will be presented at local, regional, national and international conferences. Collaborators will be encouraged to present both the protocol and the results. In addition, the results will be intermittently disseminated via publication in a peer-reviewed medical journal. All collaborators will be coauthors of resulting presentations and PubMed citable coauthors of resulting publications. The decision to submit data for publication will be agreed by the steering committee. Following publication and completion of the study, the fully anonymised dataset will be made publicly available. Hospital-level data will not be released or published on behalf of the collaborative. Collaborators will have the opportunity to undertake subanalyses of the data for their country (if all collaborators from that country agree), region or continent.

\section{Twitter Soham Bandyopadhyay @SohamBGlobal}

Acknowledgements The authors thank the University of Oxford Medical Sciences Division IT Services Systems Team for the REDCap administration and management, Lucy Davies for her statistics input and all of their collaborators from their country leads to the members of our local mini-teams for driving the study forward.

Collaborators Noel Peter; Kokila Lakhoo; Soham Bandyopadhyay; Simone Abib; Hafeez Abdelhafeez; Shaun Wilson; Max Pachl; Benjamin Martin; Sonal Nagras; Mihir Sheth; Catherine Dominic; Suraj Gandhi; Divya Parwani; Rhea Raj; Diella Munezero; Rohini Dutta; Nsimire Mulanga Roseline; Kellie McClafferty; Armin Nazari; Smrithi Sriram; Sai Pillarisetti; King-David Nweze; Aishwarya Ashwinee; Gul Kalra; Poorvaprabha Patil; Priyansh Nathani; Khushman Kaur Bhullar; Muhammed 
Elhadi; Maryam Khan; Nehal Rahim; Shweta Madhusudanan; Joshua Erhabor; Manasi Shirke; Aishah Mughal; Darica Au; Mahan Salehi; Sravani Royyuru; Mohamed Ahmed; Syeda Namayah Fatima Hussain; Daniel Robinson; Anna Casey; Mehdi Khan; Alexandre Dukundane; Kwizera Festus; Vaishnavi Govind; Rohan Pancharatnam; Lorraine Ochieng; Elliott H Taylor; Hritik Nautiyal; Marta de Andres Crespo; Somy Charuvila; Alexandra Valetopoulou

Contributors NP, SB and KL conceived the project, contributed equally to the design of the project and drafted the manuscript. The Global Health Research Group on Children's Non-Communicable Diseases Collaborative provided input into the design of the study and the content of the manuscript. All authors approved the final manuscript and are willing to take responsibility for appropriate portions of the content.

Funding The authors have not declared a specific grant for this research from any funding agency in the public, commercial or not-for-profit sectors.

Competing interests None declared.

Patient consent for publication Not required.

Provenance and peer review Not commissioned; externally peer reviewed.

Supplemental material This content has been supplied by the author(s). It has not been vetted by BMJ Publishing Group Limited (BMJ) and may not have been peer-reviewed. Any opinions or recommendations discussed are solely those of the author(s) and are not endorsed by BMJ. BMJ disclaims all liability and responsibility arising from any reliance placed on the content. Where the content includes any translated material, BMJ does not warrant the accuracy and reliability of the translations (including but not limited to local regulations, clinical guidelines, terminology, drug names and drug dosages), and is not responsible for any error and/or omissions arising from translation and adaptation or otherwise.

Open access This is an open access article distributed in accordance with the Creative Commons Attribution Non Commercial (CC BY-NC 4.0) license, which permits others to distribute, remix, adapt, build upon this work non-commercially, and license their derivative works on different terms, provided the original work is properly cited, appropriate credit is given, any changes made indicated, and the use is non-commercial. See: http://creativecommons.org/licenses/by-nc/4.0/.

\section{ORCID iD}

Soham Bandyopadhyay http://orcid.org/0000-0001-6553-3842

\section{REFERENCES}

1 The Health Foundation. COVID-19: five dimensions of impact, 2020. Available: https://www.health.org.uk/news-and-comment/blogs/ covid-19-five-dimensions-of-impact

2 Shehata IM, Elhassan A, Jung JW, et al. Elective cardiac surgery during the COVID-19 pandemic: proceed or Postpone? Best Pract Res Clin Anaesthesiol 2020;34:643-650.

3 Gaudino M, Chikwe J, Hameed I, et al. Response of cardiac surgery units to COVID-19: an Internationally-Based quantitative survey. Circulation 2020;142:300-2.

4 Cano-Valderrama O, Morales X, Ferrigni CJ, et al. Reduction in emergency surgery activity during COVID-19 pandemic in three Spanish hospitals. Br J Surg 2020;107:e239.

5 Check Hayden E. Ebola outbreak shuts down malaria-control efforts. Nature 2014;514:15-16.

6 Dong Y, Mo X, Hu Y, et al. Epidemiology of COVID-19 among children in China. Pediatrics 2020;145. doi:10.1542/peds.2020-0702. [Epub ahead of print: 1603 2020]

7 Steliarova-Foucher E, Colombet M, Ries LAG, et al. International incidence of childhood cancer, 2001-10: a population-based registry study. Lancet Oncol 2017;18:719-31.

8 Magrath I, Steliarova-Foucher E, Epelman S, et al. Paediatric cancer in low-income and middle-income countries. Lancet Oncol 2013;14:e104-16.

9 Pritchard-Jones K, Pieters R, Reaman GH, et al. Sustaining innovation and improvement in the treatment of childhood cancer: lessons from high-income countries. Lancet Oncol 2013;14:e95-103.

10 SEER Statistics. Cancer statistics review, 1975-2015. Available: https://seer.cancer.gov/archive/csr/1975_2015/

11 Bhakta N, Force LM, Allemani C, et al. Childhood cancer burden: a review of global estimates. Lancet Oncol 2019;20:e42-53.
12 Eden T. Childhood cancers in low-and middle-income countries: prevention and potentially curable treatment.

13 Ribeiro RC, Steliarova-Foucher E, Magrath I, et al. Baseline status of paediatric oncology care in ten low-income or mid-income countries receiving my child matters support: a descriptive study. Lancet Oncol 2008;9:721-9.

14 World Bank DataBank. World development indicators. Available: https://databank. worldbank.org/reports.aspx? source=2\&country= WLD

15 Howard SC, Metzger ML, Wilimas JA, et al. Childhood cancer epidemiology in low-income countries. Cancer 2008;112:461-72.

16 Gupta S, Howard SC, Hunger SP. Treating Childhood Cancer in Lowand Middle-Income Countries. In: Disease control priorities. . Third Edition. The World Bank, 2015: Volume 3. p 121-46. https://www. ncbi.nlm.nih.gov/books/NBK343626/

17 Silbermann M, Al-Hadad S, Ashraf S, et al. MECC regional initiative in pediatric palliative care. J Pediatr Hematol Oncol 2012;34:S1-11.

18 Cancer Research UK. What's happened to cancer services during the COVID-19 pandemic? Available: https://scienceblog. cancerresearchuk.org/2020/09/11/whats-happened-to-cancerservices-during-the-covid-19-pandemic/

19 ESMO. COVID-19 Pandemic Halts Cancer Care and Damages Oncologists' Wellbeing. Available: https://www.esmo.org/newsroom/ press-office/esmo2020-covid-pandemic-halts-cancer-careoncologist-wellbeing

20 St. Jude Global. Global registry of COVID-19 in pediatric cancer, 2020. Available: https://global.stjude.org/en-us/global-covid-19observatory-and-resource-center-for-childhood-cancer/registry.html

21 COSECSA. COSECSA [Internet]. [cited 2020 Oct 2]. Available from. Available: http://www.cosecsa.org/

22 Global Initiative for Children's Surgery, 2020. Available: https://www. globalchildrenssurgery.org/

23 PAPSA, 2020. Available: http://www.papsa-africa.org/

24 Siop, 2020. Available: https://siop-online.org/

25 IPSO. The International Society of paediatric surgical oncology. Available: https://ipso-online.org/

26 Mostert S, Arora RS, Arreola M, et al. Abandonment of treatment for childhood cancer: position statement of a SIOP PODC Working group. Lancet Oncol 2011;12:719-20.

27 Blencowe N, Glasbey J, Heywood N, et al. Recognising contributions to work in research Collaboratives: guidelines for standardising reporting of authorship in Collaborative research. Int J Surg 2018;52:355-60.

28 Wright NJ, Wright N, Ade-Ajayi N, Global PaedSurg Research Collaboration. Management and outcomes of gastrointestinal congenital anomalies in low, middle and high income countries: protocol for a multicentre, international, prospective cohort study. BMJ Open 2019;9:e030452.

29 Bandyopadhyay S, Shortland T, Wadanamby SW, et al. Global health education in UK medical schools (GHEMS) study protocol. J Glob Health Rep 2019;3.

30 Steliarova-Foucher E, Stiller C, Lacour B, et al. International classification of childhood cancer, third edition. Cancer 2005;103:1457-67.

31 WHO. Cancer in children. Available: https://www.who.int/news-room/ fact-sheets/detail/cancer-in-children

32 Lai A, Hoong Chang W, Lai AG. Estimating excess mortality in people with cancer and multimorbidity in the COVID-19 emergency. medRxiv 2020;15:16.

33 Maringe C, Spicer J, Morris M, et al. The impact of the COVID-19 pandemic on cancer deaths due to delays in diagnosis in England, UK: a national, population-based, modelling study. Lancet Oncol 2020;21:1023-34. doi:10.1016/S1470-2045(20)30388-0

34 Seyi-Olajide JO, Anderson JE, Kaseje N, et al. Inclusion of children's surgery in national surgical plans and child health programmes: the need and roadmap from global initiative for children's surgery. Pediatr Surg Int 2021;37:529-37. doi:10.1007/s00383-020-04813-x

35 von Elm E, Altman DG, Egger M, et al. The strengthening the reporting of observational studies in epidemiology (STROBE) statement: guidelines for reporting observational studies. J Clin Epidemiol 2008;61:344-9.

36 World Bank. World bank country and lending groups, 2021. Available: https://datahelpdesk.worldbank.org/knowledgebase/articles/906519world-bank-country-and-lending-groups

37 Gupta S, Aitken JF, Bartels U, et al. Paediatric cancer stage in population-based cancer registries: the Toronto consensus principles and guidelines. Lancet Oncol 2016;17:e163-72. 